Apidologie, 1975, 6 (4), 341-359.

\title{
ÉTUDE DU COMPORTEMENT D'ANDRENa CARANTONICA PEREZ AU COURS DE LA FLORAISON DES POMMIERS DE LA VARIÉTÉ GOLDEN DELICIOUS
}

\author{
Verhaltensstudie an Andrena carantonica Perez \\ während der Blütezeit von Golden delicious Apfelbäumen
}

\section{J. CHANSIGAUD}

Station de Recherches sur l'Abeille et les Insectes Sociaux, I.N.R.A. 91440 Bures-sur-Yvette

\section{SUMMARY}

STUDY OF THE Andrena carantonica BEHAVIOR

DURING THE BLOOMING OF THE GOLDEN DELICIOUS APPLE TREES

The author keeps Andrena carantonica Perez under greenhouse conditions in order to note the main characteristics of its nest, while studying the behavior of this bee in the environment.

The flights of $A$. carantonica take place in spring when Golden delicious apple trees are blooming. The number of wild bees is practically the same between $10^{\circ} \mathrm{C}$ and $16^{\circ} \mathrm{C}$ whereas the proportion of honey bees is much higher from $13^{\circ} \mathrm{C}$ on. More over $A$. carantonica gathers a rather important quantity of pollen from fruit trees. For example, $A$. carantonica takes part in the pollination of the apple trees in the La Minière orchard near Versailles. However, as far as pollination is concerned, the participation of wild bees will have more in fluence on the fructification, if the latter takes place at temperatures between $10^{\circ} \mathrm{C}$ and $13{ }^{\circ} \mathrm{C}$, these temperatures being detrimental to the activity of Apis mellifica $L$.

\section{RESUMÉ}

L'auteur élève en serre Andrena carantonica Perez a fin de noter les principales caractéristiques du nid de l'abeille dont il étudie par ailleurs le comportement dans le milieu naturel.

Les vols d' $A$. carantonica interviennent au printemps, au moment de la floraison des pommiers Golden delicious. Le nombre des abeilles sauvages est à peu près le même entre $10^{\circ} \mathrm{C}$ et 
$16^{\circ} \mathrm{C}$ tandis que la quantité d'abeilles domestiques est beaucoup plus élevée à partir de $13{ }^{\circ} \mathrm{C}$. De plus l'Andrène récolte une quantité relativement importante de pollen provenant des arbres fruitiers. Ainsi, Andrena carantonica participe à la pollinisation des pommiers du verger de La Minière près de Versailles. Toutefois, en matière de pollinisation, l'intervention de l'abeille sauvage aura davantage d'incidence sur la mise à fruit si celle-ci se produit à des températures oscillant entre $10^{\circ} \mathrm{C}$ et $13^{\circ} \mathrm{C}$; ces températures étant défavorables à l'activité d'Apis mellifica.

\section{INTROD UCTION}

Les abeilles sauvages que l'on rencontre en milieu rural dans les vergers de la région parisienne appartiennent pour la plupart à la famille des Andrenidae. Anasiewicz (1971) a fait la même constatation à la suite d'une série de prospections effectuées dans un verger situé près de Lublin en Pologne.

Nous avons commencé l'étude des espèces dont les temps de vol sont les plus longs par rapport à la floraison des poiriers et des pommiers. En accord avec Chambers (1946), nous pensons en effet que le synchronisme entre l'activité pollinisatrice et les dates de floraison est fonction de la durée plus ou moins longue des périodes de vol. C'est la raison pour laquelle nous avons choisi de travailler sur Andrena carantonica Perez, (synonyme d'Andrena jacobi Perkins) dont les temps de vol s'échelonnent depuis la mi avril jusqu'à la fin du mois de mai.

Le travail est basé sur l'étude de trois sujets principaux : le premier concerne la structure du nid; le second a trait à la fréquence relative des vols en fonction des variations de température; le troisième sujet se rapporte à la nature des charges de pollen d' $A$. carantonica. Dans le second thème, nous comparons au même moment le comportement de l'abeille sauvage avec celui d'Apis mellifica.

\section{I. - POSITION SYSTEMATIQUE - RÉPARTITION GÉogRAPHIQUE CYCLE ET PERIODE DE VOL - ORGANES REPRODUCTEURS}

A l'intérieur de la superfamille des Apoïdea, Andrena carantonica appartient à la famille des Andrenidæ dont le genre principal est Andrena; ce genre a été notamment décrit par Hedicke (1930) et Michener (1944).

Divers travaux mentionnent la présence d'A. carantonica en Europe occidentale, moyenne et septentrionale, (Chambers 1946, Kocourex 1966).

Dans le bassin parisien, la période de vol des abeilles commence au mois d'avril et s'achève au cours de la dernière décade du mois de mai. Les pontes interviennent au mois de mai. D'après nos élevages, le développement larvaire serait de un mois environ et l'apparition des premiers imagos interviendrait à la fin du mois d'août; ces derniers n'émergeant du sol qu'au printemps de l'année suivante. 


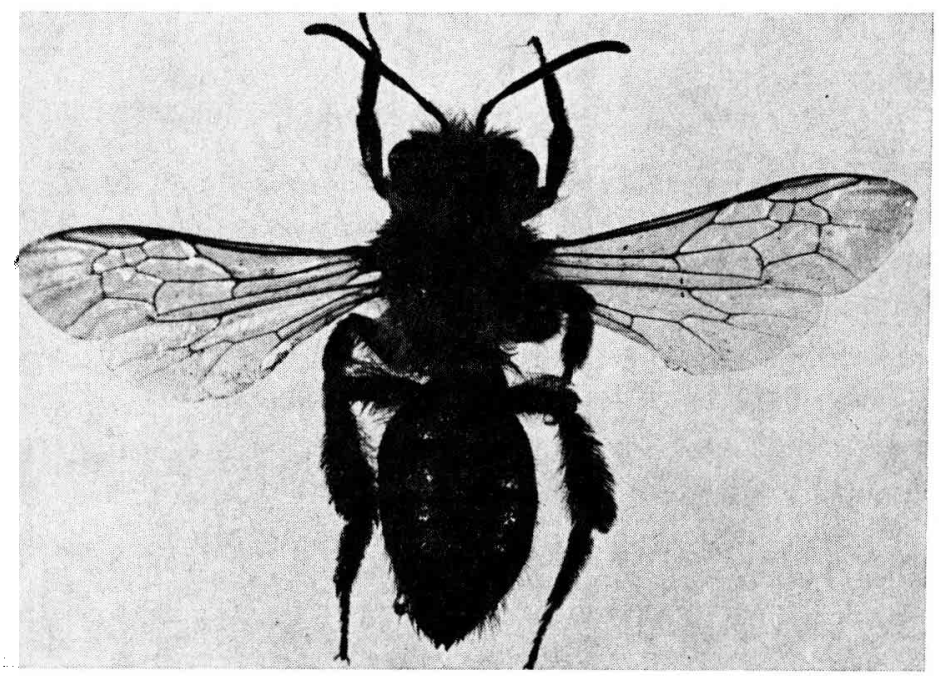

Fig. 1. - Femelle d'A. carantonica Perez. (grossissement $\times 5$ )

Aв8. 1. - Weibchen von Andrena carantonica Perez (Vergr. $\times 5$ ).

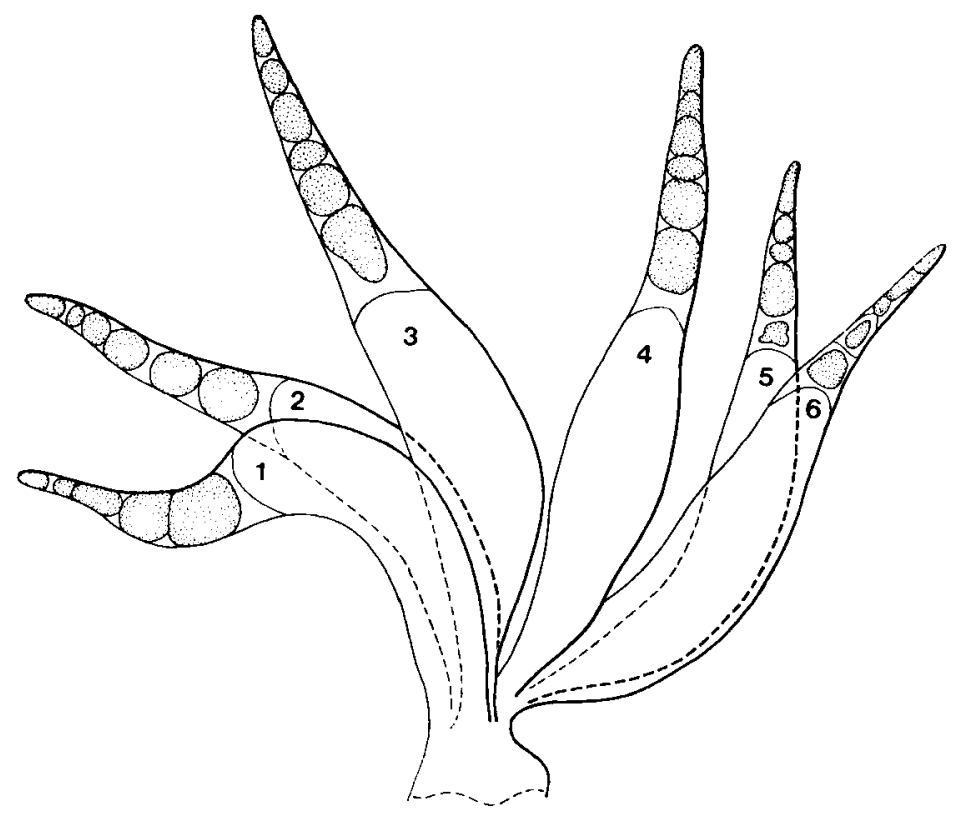

Fig. 2. - Schéma des ovaires d' $A$. carantonica, (6 ovarioles, 1-6 : œufs mûrs) ABB. 2. - Ovarien von A. carantonica (6 Ovariolen; 1-6 : reife Eier). 
Les ovaires sont au nombre de deux et chaque ovaire comporte trois ovarioles, soit six au total.

\section{II. - ÉLEVAGES}

L'étude des abeilles primitives à l'extérieur présente de sérieuses difficultés : les populations sont en général peu importantes, la découverte des sites de nidification est aléatoire et tout ce qui se passe à l'intérieur du nid demeure caché. Les biologistes ont donc cherché à élever les abeilles primitives. Le précurseur en la matière est Ove MeIDELl qui, dès 1935, construit un terrarium lui permettant d'élever certaines abeilles primitives. Plus tard, d'autres chercheurs comme Batra (1964), Steiner (1965), Stockammer (1966) mettent au point des cages adaptées à l'habitat particulier des abeilles qu'ils désirent étudier. Nous avons nous mêmes mis au point un dispositif qui permet d'obtenir la nidification d'Andrena carantonica.

\section{Matériel et méthodes}

Le 30 avril 1973, à l'aide d'un filet fauchoir, nous avons capturé douze femelles d' $A$. carantonica sur les fleurs de Taraxacum sp dans le verger de La Minière près de Versailles. Ces abeilles ont été ensuite placées dans une serre vitrée de $1,80 \mathrm{~m}$ sur $1,50 \mathrm{~m}$ et de $1 \mathrm{~m}$ de haut. Constitué d'un mélange de terre et de sable, le sol de la serre atteignait une profondeur de $50 \mathrm{~cm}$.

Pour construire leurs nids, les abeilles avaient le choix entre un limon argileux et un limon sableux. La terre destinée à la nidification des abeilles a été prélevée à l'extérieur entre $20 \mathrm{~cm}$ et $40 \mathrm{~cm}$ de profondeur; elle était ensuite transferrée dans des boîtes en bois de $15 \mathrm{~cm}$ de côté et de $40 \mathrm{~cm}$ de haut. Pour chaque type de sol nous avons constitué quatre boîtes de nidification, soit huit au total.

La boîte de nidification comprend deux parties amovibles, (Fig. 3 A). Ce dispositif permet

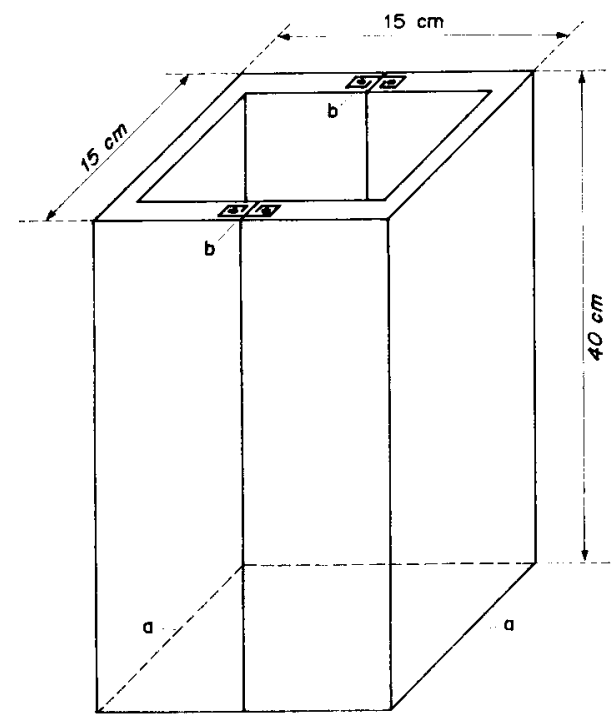

Frc. 3 A. - Boîte de nidification fermée : a) charnières d'ouverture : b) crochets de fixation des deux parties mobiles de la boîte.

ABв. 3 A. - Geschlossener Nistbehälter; a) Scharniere zum Öffnen, b) Befestigungshaken der beweglichen Teile. 
l'observation partielle du nid dans la mesure où la motte de terre conserve sa forme initiale et ne s'effrite pas : il faut donc procéder par étape, ne pas découvrir entièrement la motte de terre dont une moitié reste tour à tour solidaire d'un des éléments de la boîte, au fur et à mesure des investigations, (Fig. $3 \mathrm{~B}$ ).

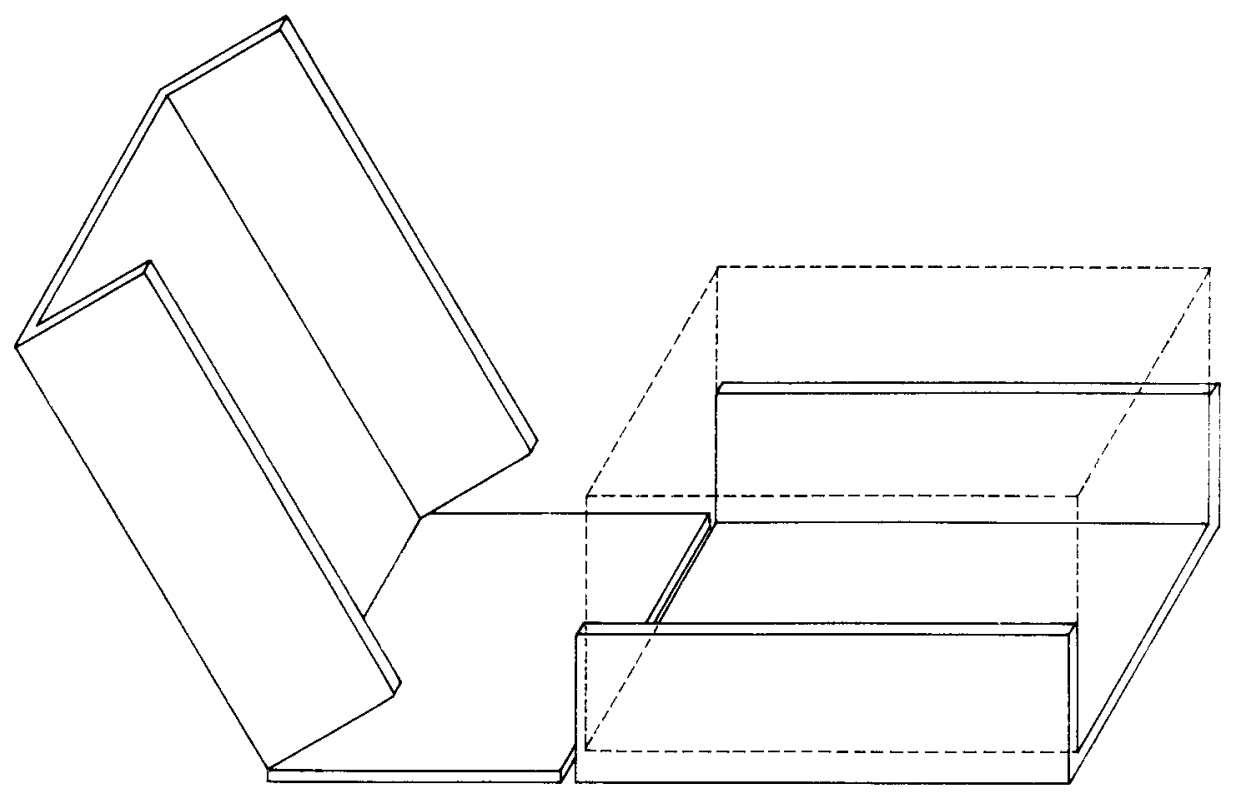

Fig. 3 B. - Boîte de nidification ouverte, (la motte de terre est représentée en pointillés).

Aвв. 3 B. - Geöffneter Behälter (der Erdklumpen ist punktiert).

L'observation partielle du nid à laquelle nous venons de faire allusion concerne le conduit principal : celui-ci se situe fréquemment sur l'un des côtés de la motte de terre; du conduit principal partent les galeries secondaires à l'extrémité desquelles aboutissent les cellules, (Fig. $3 \mathrm{C}$ ). Ces dernières ne sont pas toujours visibles, pas plus d'ailleurs que les galeries secondaires que l'on arrive à découvrir en creusant le sol à l'aide d'un grattoir.

Commencée le 30 avril, l'expérimentation s'est achevée à la fin du mois de juin. Pendant cette période, la terre des boîtes de nidification a été régulièrement humidifiée par pulvérisation d'eau à la surface du sol toutes les quarante-huit heures. Au cours de la seconde quinzaine du mois de juin, les enceintes à l'intérieur desquelles les abeilles avaient nidifié ont été transferrées successivement au laboratoire, a fin d'étudier la structure du nid conformément aux explications du paragraphe précédent. Nous avons également évalué l'humidité de la terre des boîtes de nidification : un échantillon de terre dont le poids varie de 100 à $150 \mathrm{~g}$ a été prélevé tous les dix centimètres à partir de la surface du sol de la boîte. Rappelons que l'humidité du sol s'obtient par la différence entre le poids de terre humide et le poids de terre sèche d'un échantillon qui, dans le cas présent, a séjourné pendant plusieurs jours au laboratoire à une température située entre $20^{\circ} \mathrm{C}$ et $25^{\circ} \mathrm{C}$. La valeur obtenue $\times 100$ rapportée au poids initial de l'échantillon représente le pourcentage d'humidité.

Pour obtenir la nidification des abeilles dans des zones de terre précises il est indispensable d'empêcher celles-ci de nidifier au hasard dans la serre. Lorsqu'il s'agit d'une enceinte peu volumineuse, cela ne pose pas de problèmes particuliers : il suffit de recouvrir le sol d'une dalle en 


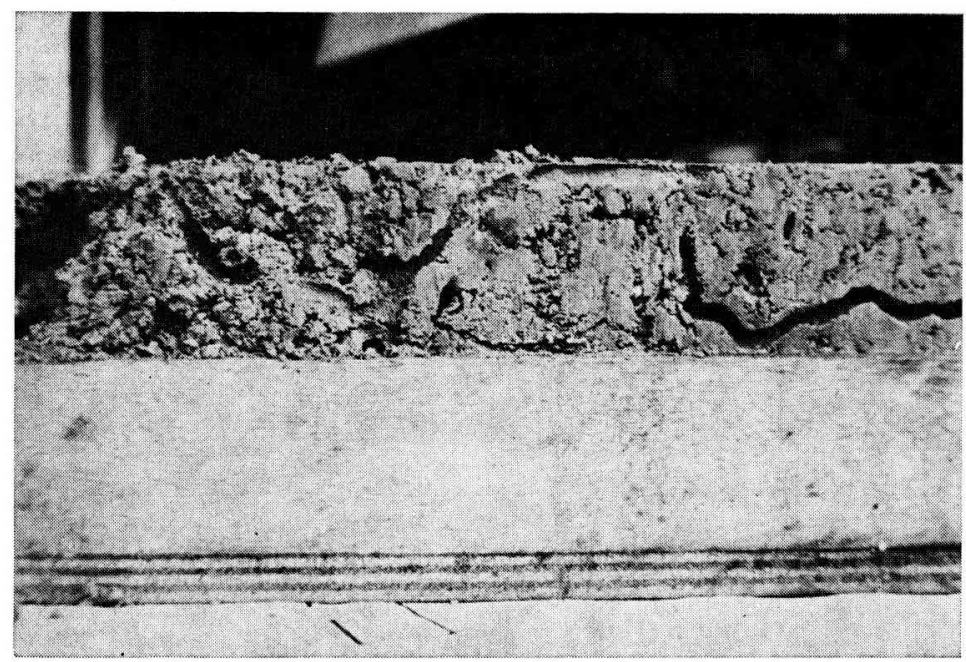

FIG. 3 C. - Boîte de nidification ouverte en position horizontale. Sur l'un des côtés de la motte de terre, tracé du conduit principal et des conduits secondaires à l'extrémité desquels nous voyons les cellules.

Авв. 3 C. - Horizontal liegender, geöffneter Nistbehälter. Auf der einen Seite des Erdklumpens der Umriss des Hauptganges und der Nebengänge, an deren Ende die Zellen zu sehen sind.

ciment par exemple sur laquelle ont été percés un certain nombre d'orifices. Chaque orifice est destiné à recevoir une boîte de nidification dont les neuf dixième de la hauteur sont insérés dans la terre, (Fig. 4).

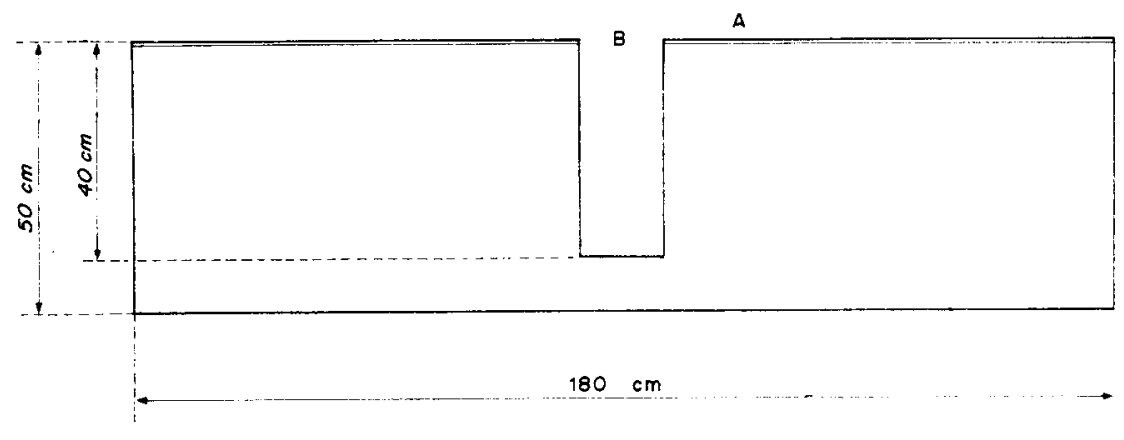

Echelle ou $1 / 9^{\circ}$

Fic. 4. - Profil de la serre où sont insérées les boîtes de nidification : dalle isolant le sol de la serre (a): b-emplacement de la boîte de nidification.

Aв8. 4. - Ansicht des Glashauses, in dem die Nistbehälter untergebracht sind : a) Isolierplatte für den Boden, b) Platz für die Nistbehälter. 
Le dispositif d'élevage que nous venons de décrire ne constitue pas un terrarium comparable à celui de MeIDELL à l'intérieur duquel les Apides sont soumis à des conditions strictement artificielles. Nos Andrènes sont en effet placées dans une serre et restent dépendantes de certains facteurs abiotiques du milieu naturel, (température et durée d'éclairement).

\section{Structure du nid}

Il est délicat d'interpréter des résultats provenant d'une cage d'élevage : l'obtention d'une construction atypique est toujours possible lorsque la vie de l'insecte s'écarte du milieu naturel. C'est pourquoi nous ne relatons ici que les caractéristiques du nid susceptibles de se reproduire dans la nature.

A partir des 12 Andrènes qui ont été récoltées, nous avons obtenu en définitive cinq nids, lesquels ont été approvisionnés en pollen : quatre ont été construits dans le limon sableux et un dans le limon argileux, (tableau 1).

Le diamètre de l'entrée du nid est de $8 \mathrm{~mm}$ et la hauteur du tumulus qui l'entoure de $20 \mathrm{~mm}$ environ, (figure 5).

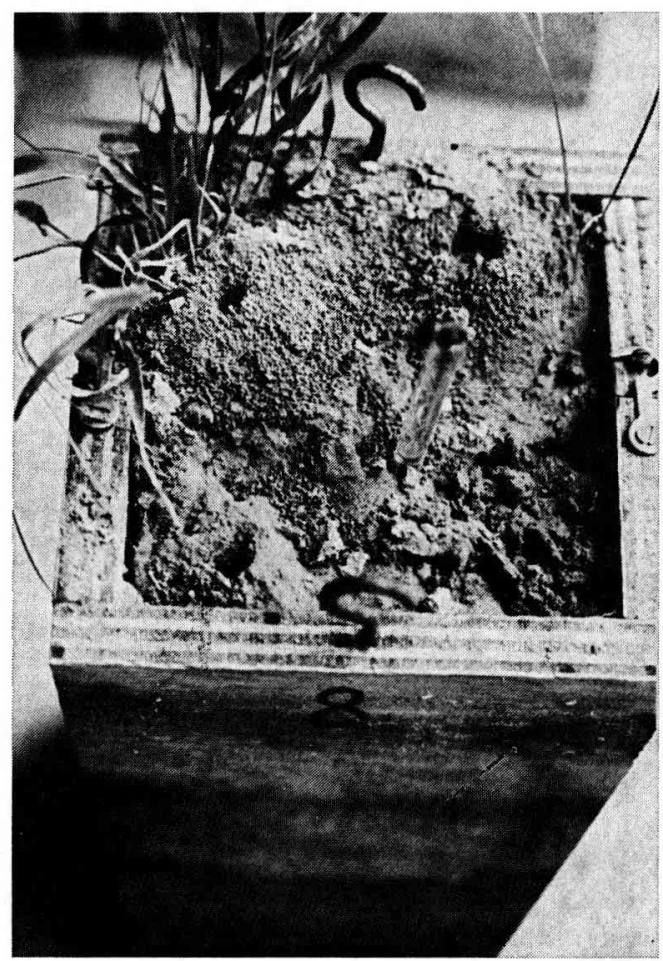

Fıg. 5. - Tumulus à la surface du sol portant en son milieu l'orifice d'entrée du nid. Авв. 5. - Erdhügel auf dem Boden; in der Mitte Eingangsöffnung des Nestes. 
Quel que soit le type de sol, le conduit principal atteint une profondeur de 20 à $30 \mathrm{~cm}$. Le diamètre du conduit principal et des galeries secondaires est de $5 \mathrm{~mm}$.

Les cellules sont généralement construites entre 15 et $30 \mathrm{~cm}$ de profondeur dans une zone où l'humidité du sol est de 18-20\% pour un limon argileux et de 11-12\% pour un limon sableux, (tableau 1).

TABL. 1. - Développement du nid d'A. carantonica Perez à l'intérieur de 3 boîtes de nidification et pourcentages d'humidité du sol à différentes profondeurs.

TAB. 1. - Entwicklung des Nestes von Andrena carantonica Perez im Innern von drei Nistbehältern; Bodenfeuchtigkeit in verschiedenen Tiefen in Prozenten.

\begin{tabular}{|c|c|c|c|c|c|c|c|}
\hline \multirow{2}{*}{$\begin{array}{c}\text { Profondeur } \\
\text { de prélèvement } \\
\text { des couches de terre } \\
\text { à l'extérieur (de ... à) } \\
\text { Höhe der } \\
\text { ausgehobenen Erde, } \\
\text { aussen, von ... bis }\end{array}$} & \multirow[t]{2}{*}{$\begin{array}{l}\text { Type de sol } \\
\text { Bodenart }\end{array}$} & \multirow[t]{2}{*}{$\begin{array}{c}\text { Nombre } \\
\text { de nids } \\
\text { Anzahl } \\
\text { Nester }\end{array}$} & \multirow{2}{*}{$\begin{array}{c}\text { Nombre } \\
\text { de cellules } \\
\text { par nid } \\
\text { ZahI der } \\
\text { Zellen je } \\
\text { Nest }\end{array}$} & \multicolumn{4}{|c|}{$\begin{array}{c}\% \text { d'humidité de la terre des boîtes } \\
\text { de nidification : prélèvement d'un } \\
\text { échantillon tous les dix centimètres } \\
\% \text { Feuchtigkeit der Erde } \\
\text { in den Nistbehältern; } \\
\text { Bodenentnahme alle } 10 \mathrm{~cm}\end{array}$} \\
\hline & & & & $10 \mathrm{~cm}$ & $20 \mathrm{~cm}$ & $30 \mathrm{~cm}$ & $40 \mathrm{~cm}$ \\
\hline de 20 à $30 \mathrm{~cm}$ & $\begin{array}{l}\text { Limon } \\
\text { Lehm }\end{array}$ & 1 & 6 & $15 \%$ & $19 \%$ & $20 \%$ & $20 \%$ \\
\hline de 20 à $30 \mathrm{~cm}$ & $\begin{array}{c}\text { Limon et sable } \\
\text { Lehm + Sand }\end{array}$ & 1 & 6 & $14 \%$ & $12 \%$ & $12 \%$ & $11 \%$ \\
\hline de 30 à $40 \mathrm{~cm}$ & $\begin{array}{c}\text { Limon et sable } \\
\text { Lehm + Sand }\end{array}$ & 3 & 19 & $11 \%$ & $12 \%$ & $11 \%$ & $12 \%$ \\
\hline
\end{tabular}

Les divers types de nids des abeilles primitives sont définis par la position des cellules dans le sol : ces dernières sont placées bout à bout, soit pédonculées, soit sessibles, soit concentrées en un rayon souvent entouré d'une cavité. Dans, le sol, la cellule du nid d'A. carantonica est en position horizontale; prolongeant la galerie secondaire, elle peut être à une distance de $20 \mathrm{~mm}$ du conduit principal, (figure 6). Il s'agit par conséquent d'une cellule pédonculée dont la longueur est de $15 \mathrm{~mm}$.

Comme chez la plupart des abeilles primitives, la cellule présente une symétrie bilatérale, (figure 7).

\section{Description de l'ceuf}

L'œuf de couleur blanche mesure entre 2,1 et $2,3 \mathrm{~mm}$, (figure 8 ); légèrement courbé en are, il est pondu à la face supérieure de la balle de pollen. 


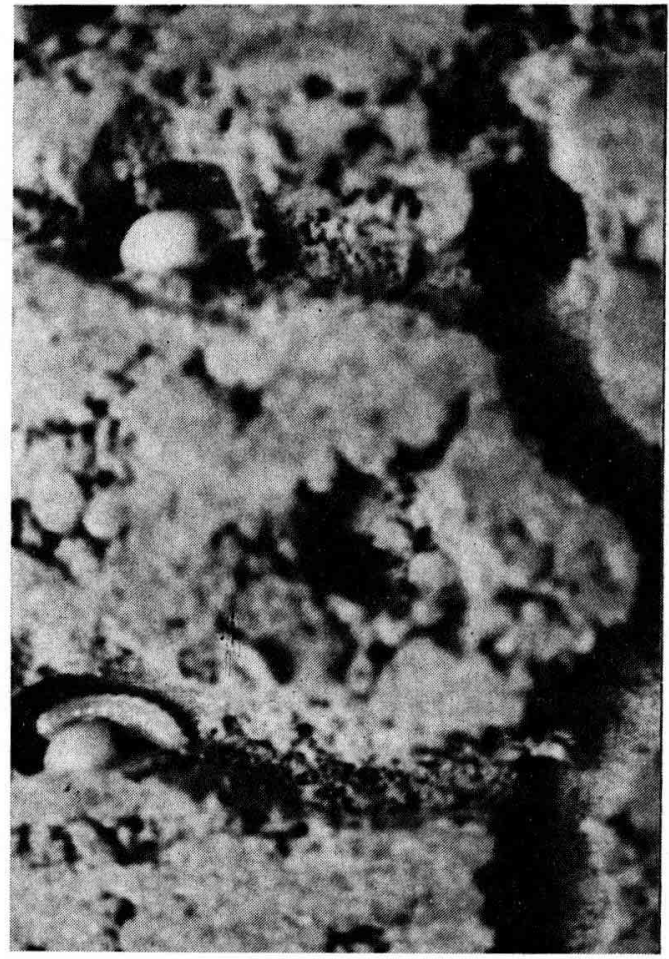

FIG. 6. - Position des cellules dans le sol : à droite le tracé du conduit principal auquel se rattachent les conduits secondaires. Chaque galerie secondaire aboutit à une cellule à l'intérieur de laquelle il y a une larve et son pain de pollen.

Aв8. 6. - Lage der Zellen im Boden : rechts Umriss des Hauptganges mit anschliessenden Nebengängen. Jeder Gang endet in einer Zelle mit einer Larve und ihrem Pollenbrot.

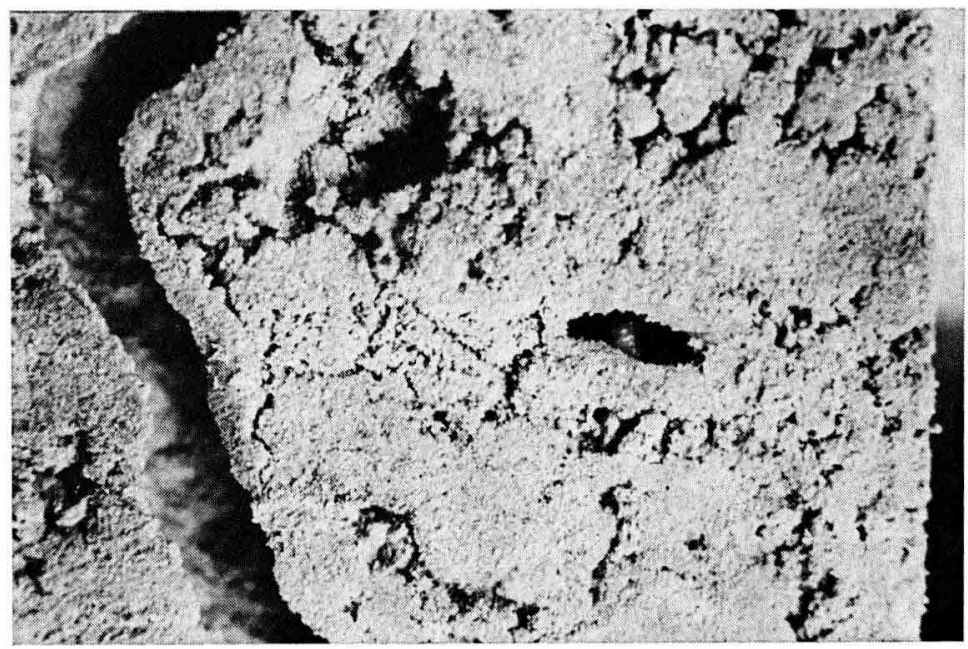

FIG. 7. - Profil d'une cellule à symétrie bilatérale.

Авв. 7. - Ansicht einer zweiseitig-symmetrischen Zelle. 


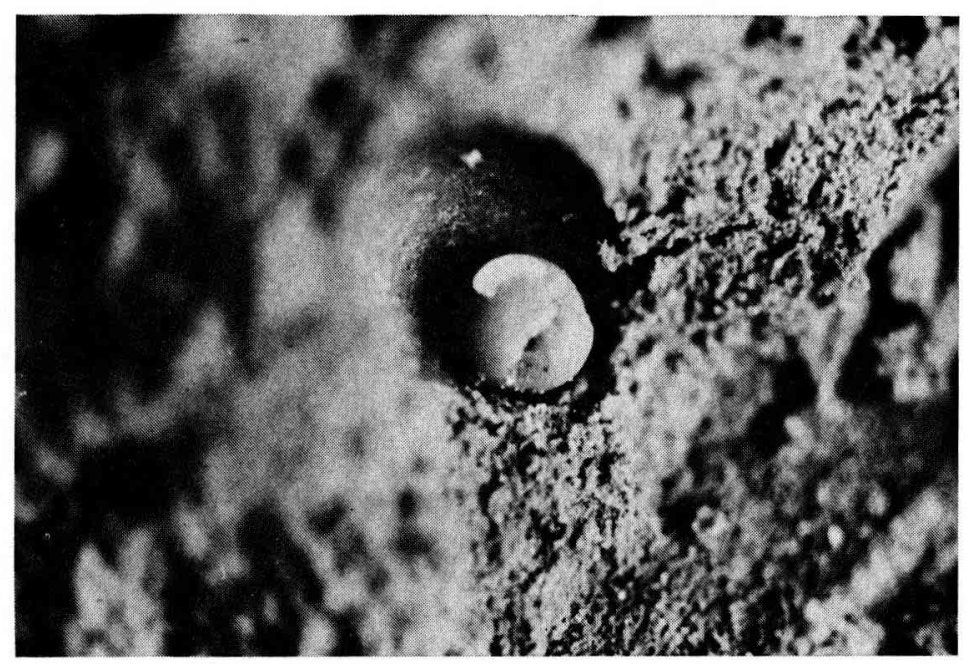

Fig. 8. - Euf déposé sur la balle de pollen.

Aв8. 8. - Auf einem Pollenklumpen abgelegtes Ei.

\section{Durée de développement}

La température moyenne hebdomadaire de la serre pour la période du 30 avril au 15 juin a été de $18^{\circ} \mathrm{C},\left( \pm 1^{\circ} \mathrm{C}\right)$.

Le 11 juin, nous avons transferré de la serre au laboratoire une boîte de nidification dans le but d'observer l'état de croissance des larves. Parmi les larves que nous avons retirées de leurs cellules, certaines étaient en fin de développement. Élevées au laboratoire à l'obscurité ces dernières ont pris le stade prénymphal à la fin du mois de juin et au début du mois de juillet. Les nymphes sont apparues à la fin du mois de juillet et au début du mois d'août. Nous avons obtenu des imagos à la fin du mois d'août, soit cent jours environ après les premières pontes qui ont eu lieu au cours de la seconde quinzaine du mois de mai. Étant donné qu'il s'agit vraisemblablement d'une espèce univoltine ce résultat laisse supposer que l'insecte hiverne à l'état adulte dans les conditions naturelles.

\section{III. - Fréquence Relative des vols d'A. Carantonica EN FONCTION DES VARIATIONS DE TEMPÉRATURE}

Les abeilles sauvages que l'on rencontre au printemps sur les fleurs des 
arbres fruitiers ont habituellement un seuil thermique d'activité inférieur à celui de l'abeille domestique. Cette constatation a été faite notamment par Bohart (1952). Ainsi, l'activité d' $A$. carantonica est moins perturbée que celle d'Apis mellifica à des températures inférieures à $13{ }^{\circ} \mathrm{C}$, (températures moyennes relevées à $2 \mathrm{M}$ sous abri).

Sans préjuger de la valeur pollinisatrice de l'abeille, le fait mérite d'être souligné au moment où les spécialistes s'accordent à reconnaître qu'une pollinisation précoce est indispensable pour la mise à fruit, lorsque l'année est froide, compte tenu du développement ovarien de la fleur, (W. H. HEYDECKER, 1972).

\section{Matériel et méthodes}

Les observations ont été faites en 1971 dans le verger de La Minière près de Versailles sur des pommiers en fleurs de la variété Golden delicious. Isolé au milieu d'une plaine où dominent les cultures céréalières, ce verger constitue l'une des principales sources de pollen pour les Apides sauvages se multipliant au printemps dans ce biotope.

Les dénombrements d'abeilles ont été entrepris du 30 avril au 5 mai; ils coïncident avec le début et la fin de la floraison des pommiers. Chaque jour, le matin de 10 à $11 \mathrm{~h}$, l'après-midi pendant une heure, entre 13 et $15 \mathrm{~h}$, nous comptions les abeilles domestiques et sauvages présentes sur les fleurs.

Contrairement aux deux années précédentes, les comptages n'ont pas été faits sur l'ensemble du verger mais uniquement sur les six premières haies fruitières de la partie sud du verger. Pendant une heure, nous nous sommes déplacés le long des rangées d'arbres, sans jamais repasser à la même place; le laps de temps nécessaire aux observations de chaque rang d'arbres étant de dix minutes. La surface que nous avons ainsi prospectée représente le cinquième de la surface totale du verger qui est de deux hectares. Nous n'avons pas effectué de captures systêmatiques d'insectes, excepté pour $A$. carantonica : quelques individus ont été capturés pour être déterminés au laboratoire. La méthode des comptages est donc visuelle : Apis mellifica et Andrena carantonica sont les seules espèces qui ont été déterminées sur-le-champ. Nous avons, $\mathrm{p}$ ar conséquent, entrepris au même moment trois comptages distincts : les deux premiers concernent respectivement $A$. mellifica et les abeilles sauvages en général, (sauf $A$. carantonica); le troisième comptage étant réservé aux individus de cette dernière espèce, (tableau 2).

Comme nous l'indiquions dans une précédente publication, cette méthode a l'avantage de donner un meilleur éventail des espèces présentes étant donné la prolongation des observations pendant une heure. Par contre, il est certain que sur un laps de temps aussi long, les chances de recenser plus d'une fois un insecte ne sont pas négligeables. C'est d'ailleurs la raison pour laquelle on effectue les comptages sur une période plus courte. De ce fait, les effectifs ne peuvent être considérés comme absolus. Nous utilisons donc le mot fréquence pour désigner la quantité d'abeilles observées car, dans le cas présent, ce terme intègre un facteur mobilité des insectes qui favorise l'abeille domestique plus mobile que les abeilles solitaires. Ainsi, d'après les dénombrements effectués pendant une heure, le matin et l'après-midi, nous pouvons connaître la fréquence relative des abeilles appartenant à l'une des espèces ci-dessus par rapport au total des observations, (pour l'ensemble des abeilles), pendant le même temps et sur le même parcours.

\section{Répartition des populations d'abeilles sauvages}

Dans la partie du verger que nous avons prospectée, la population des abeilles sauvages y compris celle d' $A$. carantonica représente en fin de matinée $34,5 \%$ et en début d'après-midi $21,2 \%$ du total des Apides dénombrés, (oolonnes $6+7$ du tableau 2). Parmi les abeilles sauvages, la population 


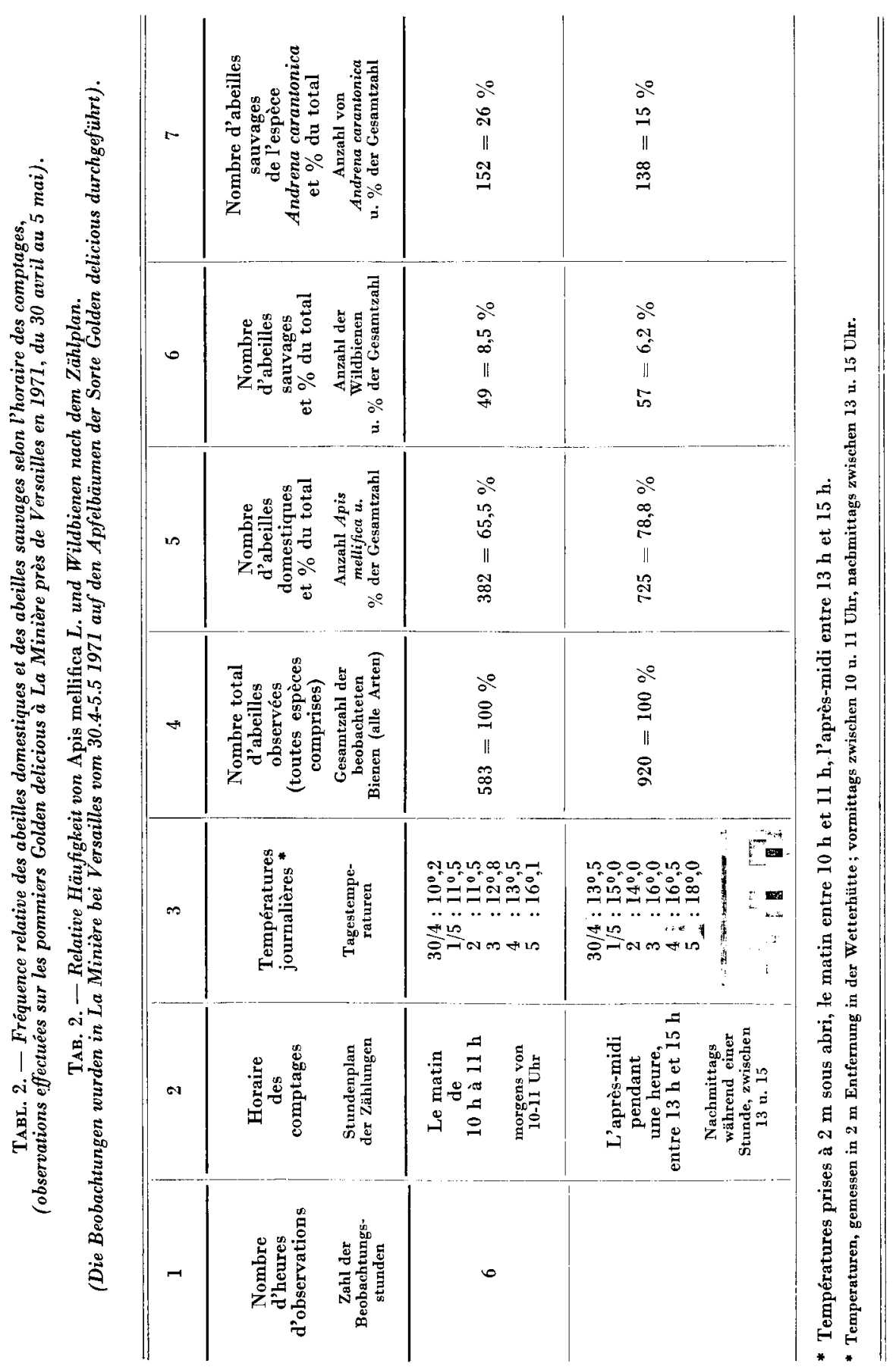


$\mathrm{d}^{\prime} A$. carantonica est la plus importante : elle représente selon l'horaire des comptages $26 \%$ puis $15 \%$ du total des pollinisateurs dénombrés.

\section{Activité de vol d'A. carantonica et d'Apis mellifica}

Le nombre des pollinisateurs sauvages, notamment celui des abeilles de l'espèce $A$. carantonica reste relativement stable au cours de la journée tandis que la fréquence relative des vols de l'abeille domestique est beaucoup plus élevée l'après-midi par rapport à la matinée, (tableau 2, colonnes 5-6-7).

Dans le but de comparer le comportement d'A. carantonica avec celui d'Apis mellifica principal pollinisateur des arbres fruitiers, rappelons que l'activité de vol des abeilles domestiques est faible à une température inférieure à $13^{\circ} \mathrm{C}$, (LANGridge 1970). Ainsi, au cours de la floraison, l'augmentation du nombre des abeilles domestiques entre 10 et $11 \mathrm{~h}$ est due à l'élévation graduelle de la température : nous observons une corrélation très positive entre les deux paramètres, (tableau 3). L'accroissement de la population des abeilles domestiques intervient surtout à partir de $13^{\circ} \mathrm{C}$; la fréquence relative des vols variant du simple au double entre $12,8^{\circ} \mathrm{C}$ et $13,5^{\circ} \mathrm{C}$, (tableau 3 ). Par contre, il n'y a pas de relation de cause à effet en ce qui concerne la population d' $A$. carantonica dont le nombre d'individus est à peu près constant de $10^{\circ} \mathrm{C}$ à $16^{\circ} \mathrm{C}$, (tableau 3 ).

\section{IV. - Nature des Charges de POLlen D'A. CARANTonica}

Les abeilles solitaires ou sociales ne visitent pas n'importe quelle fleur : il existe une spécificité alimentaire. Mais, très peu d'abeilles récoltent le pollen sur une seule plante; la majorité d'entre-elles visite un grand nombre de fleurs appartenant à différentes espèces végétales. Cependant, en présence de plusieurs sources de pollen, certaines abeilles dites oligolectiques fréquentent un nombre limité de plantes. Ainsi, à partir d'une étude des charges de pollen, Chambers (1946) a mis en évidence la préférence d'Andrena varians Kirby et d'Andrena hamorrhoa Fabricius pour le pollen des arbres fruitiers.

Les espèces oligolectiques ont généralement une adaptation à la plante hôte : l'activité des abeilles est plus ou moins synchronisée avec la floraison des plantes à proximité desquelles elles nidifient. Andrena carantonica paraît à ce sujet bien adaptée au cycle végétatif des pommiers du verger de La Minière. Aussi avons nous jugé intéressant de tester le degré de spécificité alimentaire de l'abeille sauvage à l'intérieur de ce biotope.

\section{Matériel et méthodes}

Les charges de pollen que nous avons analysées proviennent d'insectes capturés sur les fleurs des pommiers les 6 et 7 mai; c'est-à-dire tout à fait en fin de floraison. Trois fois par jour, 


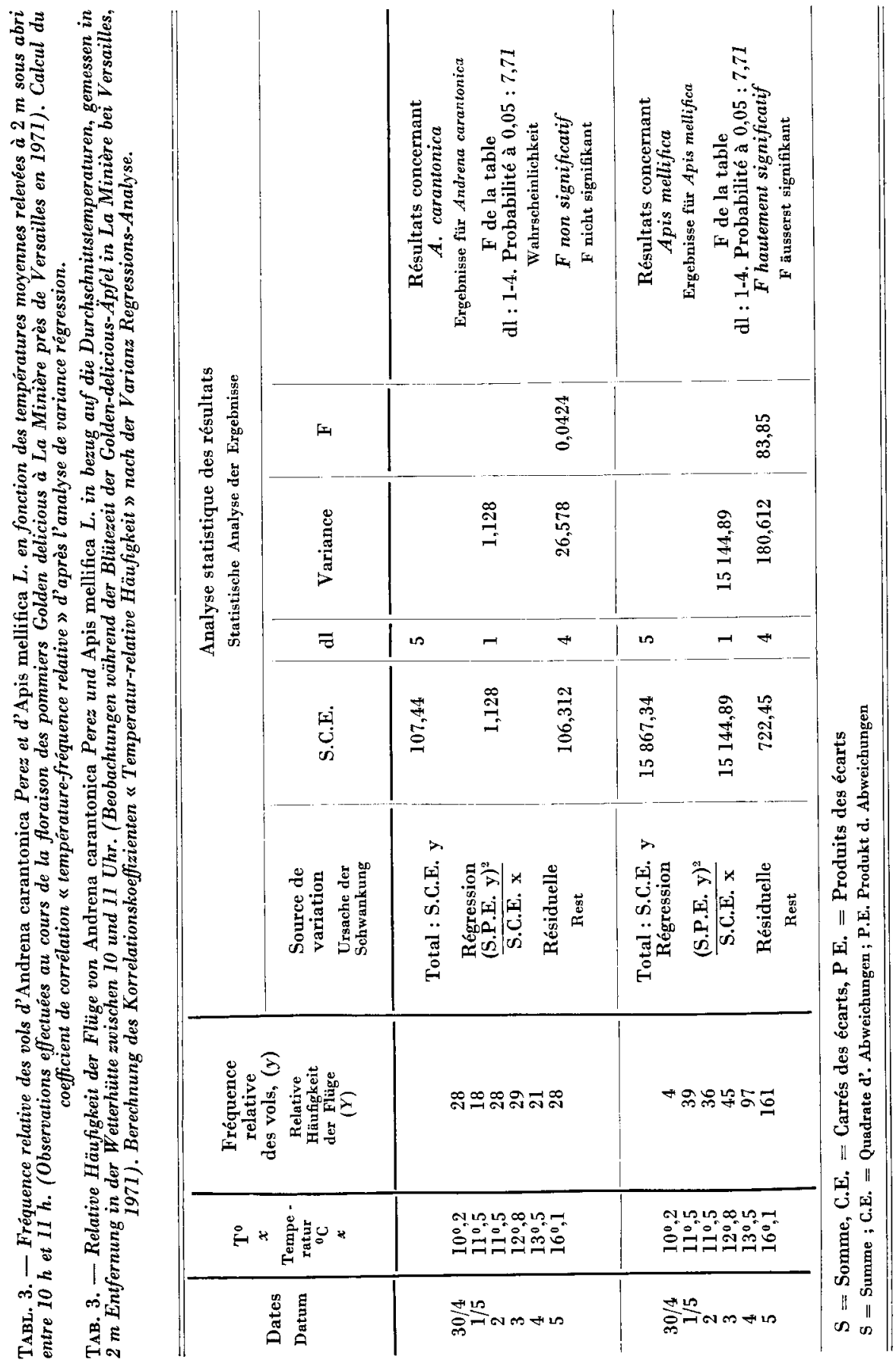




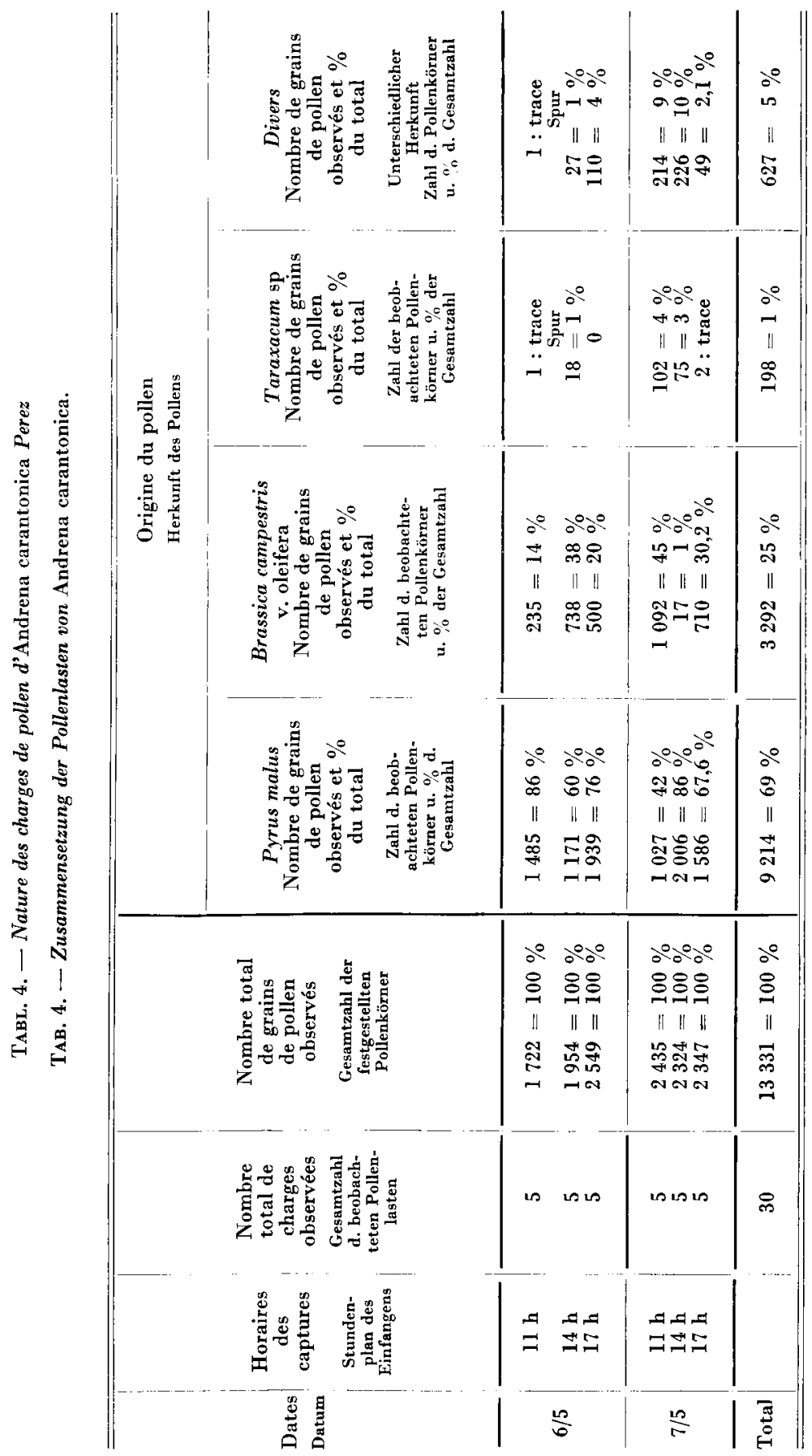


le matin à $11 \mathrm{~h}$, l'après-midi à $14 \mathrm{~h}$ et $17 \mathrm{~h}$, nous récoltions chaque fois cinq abeilles au hasard du verger, soit 15 abeilles par jour. Nous avons ainsi prélevé 30 insectes pour les deux journées consécutives. Les Apides étaient ensuite tués au cyanure et les charges de pollen retirées des organes collecteurs.

La méthode de préparation des pollens est celle qui est préconisée par Louveaux et MaURIzio (1970) : les charges de pollen sont mises en suspension dans de l'eau distillée. Quelques gouttes de la suspension sont ensuite prélevées puis placées à la surface d'une lame sur laquelle le pollen adhère lorsque le liquide est évaporé. L'élimination des substances lipidiques du pollen s'effectue par une série de lavages à l'alcool $100^{\circ}$ et à l'éther éthylique. Une goutte de glycérinegélatine est ensuite déposée sur la préparation que l'on recouvre d'une lamelle. L'étude d'une telle préparation porte en moyenne sur plus de 400 grains de pollen.

Les différentes provenances du pollen sont indiquées dans le tableau 4. Les fleurs d'adventices ou de plantes cultivées environnantes étaient surtout constituées mises à part celles des pommiers, de pissenlits et de colzas au même stade phénologique que les arbres fruitiers. Nous avons regroupé les pollens peu nombreux provenant de diverses sources, (Chêne, Bourse à pasteur...), dans la dernière colonne du tableau 4.

\section{Résultats}

Il est intéressant de constater que les récoltes de pollen se répartissent essentiellement entre les pommiers et les colzas, (tableau 4). Or, ces derniers sont environ à un kilomètre du verger tandis que les Taraxacum en fleurs sont au voisinage immédiate des arbres fruitiers. Il y a donc de la part de l'abeille sauvage une préférence marquée pour le pollen des fleurs des pommiers et des colzas.

\section{CONCLUSION}

La nidification d'A. carantonica en serre intervient dans un limon argileux ou un limon sableux. Cependant, ce dernier semble plus propice à la nidification lorsque la terre est prélevée sur le terrain à une profondeur de 30 à $40 \mathrm{~cm}$.

L'étude du comportement d'A. carantonica au printemps pendant la floraison des arbres fruitiers fait apparaître, d'une part une activité de vol inférieure à celle de l'abeille domestique, d'autre part un degré certain de spécificité alimentaire. Ce dernier critère indique que l'abeille sauvage participe à la pollinisation des pommiers du verger de La Minière. Toutefois, l'activité pollinisatrice de l'Andrène n'intervient pas immédiatement après l'émergence : la femelle s'alimente sur les fleurs pendant quelques jours et ce n'est vraisemblablement qu'à partir d'un certain stade de développement ovocytaire qu'elle commence à construire son nid et à récolter du pollen. De ce fait, la participation de l'abeille à la pollinisation sera plus efficace si l'état de maturité auquel nous venons de faire allusion coïncide avec le début et la pleine floraison, au moment où précisément les températures peuvent être inférieures à $13{ }^{\circ} \mathrm{C}$; ces températures étant défavorables à l'activité d'Apis mellifica. 


\section{REMERCIEMENTS}

Nous remercions Madame Roger Sylviane du laboratoire de Bures-sur-Yvette qui a bien voulu se charger de la détermination des pollens.

\section{ZUSAMMENFASSUNG}

Die Wildbienen, denen man im ländlichen Milieu des Pariser Gebietes begegnet, gehören zumeist zur Familie der Andrenidae. ANasiewicz (1971) hat im Verlauf einer Reihe von Untersuchungen in einer Obstanlage in der Nähe von Lublin/Polen die gleiche Beobachtung gemacht.

Wir haben unsere Untersuchungen mit den Arten begonnen, deren Flugzeiten im Verhältnis zur Blütezeit der Birnen- und Apfelbäume am längsten dauern. In Übereinstimmung mit Снамвеrs (1946) sind wir tatsächlich der Ansicht, dass der Synchronismus von Bestäubungstätigkeit und Blütezeiten eine Funktion der mehr oder weniger lange währenden Flugperioden ist. Aus diesem Grunde haben wir Andrena carantonica Perez (Synonym Andrena jacobi Perkins), deren Flugzeit sich von Mitte April bis Ende Mai erstreckt, für unsere Arbeit ausgewählt.

Die Arbeit gründet sich auf die Untersuchung dreier Hauptfragen : die erste betrifft die Nestanlage, die zweite bezieht sich auf die relative Häufigkeit der Flüge als Folge von Temperaturschwankungen, die dritte befasst sich mit der Zusammensetzung der Pollenlasten von Andrena carantonica. Im zweiten Fragenkomplex wird das Verhalten der Wildbienen mit dem von Apis mellifica $\mathrm{L}$. zum gleichen Zeitpunkt verglichen.

Das Ziel der im Glashaus durchgeführten Aufzuchten war die Erforschung der Nestanlage dieser Biene. Eine Erläuterung der Ergebnisse ist jedoch recht schwierig, da es jederzeit möglich ist, eine atypische Nestkonstruktion anzutreffen, wenn sich das Leben des Insekts ausserhalb des natürlichen Milieus abspielt. Deshalb berichten wir hier nur von Nesteigenschaften, wie sie vermutlich in der freien Natur ebenfalls vorkommen.

Beim Nestbau hatten die Bienen die Wahl zwischen einem tonigen und einem sandigen Lehm. Der Nestbau erfolgt in beiden Böden, doch wird der sandige Lehm von den Bienen bevorzugt, da die Erde $30-40 \mathrm{~cm}$ ausgehoben wird.

Wie auch der Boden beschaffen sein mag, der Hauptgang erreicht eine Tiefe von $20-30 \mathrm{~cm}$. Im allgemeinen sind die Zellen bei $18-20 \%$ Bodenfeuchtigkeit im tonigen Lehm und 11-12\% Bodenfeuchte im sandigen Lehm zwischen 15 und $30 \mathrm{~cm}$ lang.

Die verschiedenen Nesttypen der primitiven Bienen werden durch die Lage der Zellen im Boden bestimmt : sie stossen aneinander, sind gestielt oder ungestielt, manchmal zu einer Wabe zusammengefasst und von einer Grube umgeben.

Die Brutzelle von $A$. carantonica liegt waagerecht in der Erde; bei Verlängern des Nebenganges kann sie $20 \mathrm{~mm}$ vom Hauptgang entfernt sein (Abb. 6). Folglich handelt es sich um eine gestielte Zelle von $15 \mathrm{~mm}$ Länge.

Mit beendeter Entwicklung haben sich Larven, die wir vom Glashaus ins Labor gebracht haben, gegen Ende Juli/Anfang August verpuppt. Die Imagines schlüpften Ende August oder Anfang September. Da es sich um eine Art handelt, die nur eine Generation im Jahr hervorbringt, liegt die Vermutung nahe, dass das Insekt unter natürlichen Bedingungen als Imago überwintert.

Die Flüge von $A$. carantonica erfolgen im Frühling zur Blütezeit der Golden deliciousApfelbäume. Bei Temperaturen zwischen $10^{\circ} \mathrm{C}$ und $16{ }^{\circ} \mathrm{C}$ kann die Zahl der fliegenden Wild- 
bienen als konstant angesehen werden. Dagegen nimmt die Zahl der fliegenden Individuen von Apis mellifica bei Temperaturen über $13{ }^{\circ} \mathrm{C}$ stark zu. (Tab. 2 u. 3 ; s. 8 u. 9). Ausserdem sammelt Andrena eine verhältnismässig bedeutende Menge Pollen aus den Obstblüten. Der Pollen wird hauptsächlich aus Apfelblüten und aus Raps geerntet, obwohl die Rapsfelder etwa 1 km von der Obstanlage entfernt sind, während in unmittelbarer Nähe der Löwenzahn blüht. In diesem Biotop zeigt die Wildbiene eine ausgesprochene Vorliebe für Obst- und Rapspollen.

Die Untersuchungen des Verhaltens von Andrena carantonica machen deutlich, dass es sich einerseits um eine geringere Flugtätigkeit als bei Apis mellifica handelt, andererseits um einen gewissen Grad von Besonderheit der Ernährung. Dies letzte Kriterium deutet daraufhin, dass Andrena an der Bestäubung der Apfelblüten in der Obstanlage von La Minière bei Versailles beteiligt ist. Allerdings setzt die Pollensammeltätigkeit nicht unmittelbar nach dem Schlüpfen ein. Das Weibchen ernährt sich zunächst ein paar Tage auf den Blüten, und wahrscheinlich beginnt es erst mit Erreichen eines gewissen Stadiums der Ovarentwicklung mit dem Nestbau und dem Pollensammeln. Aus diesem Grund wird der Anteil dieser Bienen an der Bestäubung wirksamer sein, wenn der angesprochene Reifezustand mit dem Beginn der vollen Blüte zusammenfällt, zu einer Zeit also eintritt, wo die Temperaturen weniger als $13{ }^{\circ} \mathrm{C}$ betragen können und somit für die Tätigkeit von Apis mellifica ungünstig sind.

\section{RÉFÉRENCES BIBLIOGRAPHIQUES}

Avasiewicz, 1971, Apoïdea Hymenoptere visiting blossom of some fruit trees and black currant. Polskie. Pismo. Entomol., t 42, 2-491-506.

Batra S.W.T., 1964, Behavior of the social bee, Lasioglossum zephyrum, (Hymenoptera : Halictidae). Ins. Soc., 11, 159-185.

Batra S.W.T., 1968, Behavior of some social and solitary bees within their nests. $J$. Kansas. Ent. Soc., 41 (1), 120-133.

Brittain W.H., Newton an, 1933, A study in relative constancy of hive bees and wild bees in pollen gathering. Canad. J. Res, 9 (4), 334-349.

Bohart G.E., 1952, Pollination by native insects. Yearb. Agric. U.S. Dep. Agric., 107-121.

Chambers WH., 1946, An examination of the pollen loads of Andrena the species that visit fruit trees. J. animal. Ecol., 15, 9-21.

Dingemans-Bakels F.N., 1972, The pollen collecting activities of some andrenide bees. Extr. de Zavl. Meded .Rijksmus nat. Hist. Leiden 1972, 47, 465-467.

Hedicke H. 1930, Apoidea. Die Tierwelt Mittel-Europas, t, 5. 175-200, 1930.

Heydecker W., 1972, La pollinisation précoce est indispensable quand l'année est froide. Extr. Rev. Fr. Apicult. 1972, no 295.

KNERER G., 1969 c, Synergistic evolution of halictine nest architecture and social behavior. Canad. J. ZooL, 47 (5), 925-930.

Kocounek M. 1966, Prodromus der Hymenopteren der Tschekoslowakei Acta. faun. entomol. Musei nat. Pragae., 12, Sup. 2. Édit 30.VII. 1966.

LANGRidge D.F., Jenkins P.T., 1970, Role of honey bees in pollination of apples. Austr. Journ. of Exper. Agric. and Animal Husbandry; 10, Juin 1970.

Louveaux J., Maurizio A., Vorwohl G., 1970, Internationale Kommission für Bienenbotanik der I.U.B.S. Methodik der Melissopalynologie. Apidologie, 1970, 1 (2), 193-209.

MaLyshev, 1936, The nesting habits of solitary bees. E.O.S. 11.201-309.

Meidell O., 1958, A terrarium to investigate solitary and parasitic bees, (Hymenoptera Apidae in a artificial climate). Zoological laboratory University. Oslo. Norway. Univer. Bergen. Arb. natur. R. 5, 16 p. 1958.

Michenen C.D., 1944, Comparative external morphology phylogeny and a classification of the bees. Bull. Mus. N.H. 82 fasc. 6, 151-326. 
Osgood, E.A., 1972, Soil characteristics of nesting sites of solitary bees associated with lowbusch blueberry in Maine Orono, (Mainte U.S.A.). The life sciences and Agricul. Exp. Station. Univer of Maine 1972.8 pages.

Steiner A.L., 1965, Mise au point d'une technique d'élevage d'Hyménoptères fouisseurs en laboratoire. Bull. Soc. Ent. Fr., 70, 12-19.

Stockhammer K. A., 1967. Nesting habits and life cycle of a sweat bee Augochlora pura (Hymenoptera : Halictidae). J. Kans. Ent. Soc., 39, (n $\left.{ }^{\circ} 2\right)$, 157-192.

Stockhammer K. A., 1967, Some notes on the biology of the blue sweat bee Lasioglossum coeruleum (Apoïdea : Halictidae) J. Kans. Ent. Soc., 40 (2), 177-189. 